\title{
Testing for seasonality *
}

\author{
Philip Hans Franses \\ Econometric Institute, Erasmus University, Rotterdam, Netherlands
}

Received 7 November 1991

Accepted 17 January 1992

This paper proposes a general-to-simple test procedure for the presence of seasonal patterns in time series, which is based on tests for parameter restrictions in a general periodic model. The method is illustrated for the U.K. stock price index and the U.S. CLI index.

\section{Introduction}

Many seasonally observed economic time series such as quarterly and monthly time series show seasonally fluctuating patterns. Sometimes these patterns can be caused by holidays or festivals, and marked seasonality in several economic series is therefore quite common. There are, however, time series for which such patterns would not be easy to explain. Examples are exchange rates, stock prices and seasonally adjusted series like, e.g., the U.S. CLI index. Recent empirical evidence of such an example is reported in Canova (1989), where it is found that there appears to be seasonality in the profits from speculation in foreign exchange markets. Furthermore, Ghysels (1991) shows that the cyclical behavior of the CLI index is related to its seasonal movements. This suggests that it might be important to test for seasonality in time series.

In section 2, the present paper proposes a model-based test procedure for that purpose. In section 3 , it is applied to the quarterly time series of the U.K. stock price index and the U.S. CLI index. Section 4 concludes.

\section{A general-to-simple test procedure}

Consider a time series $y_{t}$ that is measured $s$ times per year, $t=1, \ldots, s n$, with $n$ denoting the number of years. Although there does not seem to exist a commonly accepted definition of seasonality, I will consider a time series to show seasonal fluctuations when the means and/or variances vary over $s$, and/or when $y_{t}$ can be described by $s$ different univariate time series models. For these models it may apply that the model orders and/or the values of their parameters can be different.

Correspondence to: Philip Hans Franses, Econometric Institute, Erasmus University Rotterdam, P.O. Box 1738, NL-3000 DR Rotterdam, The Netherlands.

* Thanks are due to Eric Ghysels and Denise Osborn for making available the data series. 
Given the $s$ possible different characteristics, it seems most convenient to start with a general model that allows for all these, to check this model for its adequacy, and to test for parameter restrictions which are related to seasonal behavior. Since economic time series often show nonstationary patterns, this model should also be flexible enough to incorporate such (non-)seasonal nonstationarity. $\Lambda$ vector autoregressive (VAR) model for the vector containing the observations per season, see, e.g., Franses (1990) and Osborn (1991), seems to be appropriate for this purpose. This general model is represented by

$$
q_{t}=\Pi_{1} q_{t-1}+\ldots+\Pi_{p} q_{t-p}+\mu+\omega_{t},
$$

which is a $p$ th order autoregressive model for $q_{t}^{\prime}=\left(q_{1 t}, q_{2 t}, \ldots, q_{s t}\right)^{\prime}$, i.e. the vector consisting of the $n$ observations per season $s$ on $y_{t}$, where now $t$ runs from 1 to $n$. The vectors $\mu$ and $\omega_{t}$ are $(s \times 1)$-vectors and the $\Pi_{i}$ are $(s \times s)$ parameter matrices. For convenience it is assumed that $\omega_{t} \sim \mathrm{N}_{s}(0, \Lambda)$. The $s$ different mass means and variances are reflected by these $\mu$ and $\omega_{t}$, while the $s$ models and their parameters are represented by the $\Pi_{i}$.

Given that economic time series can show nonstationary patterns that require the use of a filter like $\Delta_{1}$ or $\Delta_{s}$, where $\Delta_{k}$ is defined by $\Delta_{k} y_{t}=y_{t-k}$, the first step in the test procedure is to check which filter may be appropriate for the time series $y_{t}$. For this purpose, the method developed in Franses (1990) seems suitable. It amounts to an application of the Johansen and Juselius (1990) cointegration approach to model (1), i.e. an investigation into the number of, and restrictions on, cointegration relations between the elements of $q_{r}$. When these relations are such that either of the filters $\Delta_{1}$ or $\Delta_{s}$ can be applied, one can proceed to the next step. On the other hand, where there appear to be periodic error correcting variables that reflect equilibrium relations between the elements of $q_{t}$, one has found evidence for the presence of seasonal fluctuations.

The second step is to check whether a univariate autoregressive time series model of order $k-1$ for the possibly transformed $y_{t}$ series, say $x_{t}$, applies to all seasons $s$. Or, whether for

$$
x_{t}=\sum_{i=1}^{s} \delta_{i} D_{i t}+\sum_{i=1}^{s} \alpha_{1 i} D_{i t} x_{t-1}+\ldots+\sum_{i=1}^{s} \alpha_{k-1, i} D_{i t} x_{t-k+1}+\epsilon_{t}
$$

where the $D_{i t}$ are seasonal dummy variables, the restrictions in the hypothesis

$$
\mathrm{H}_{\mathrm{T}}: \delta_{s}=\delta, \quad \alpha_{1 s}=\alpha_{s}, \ldots, \alpha_{k-1, s}=\alpha_{k-1}
$$

for all $s$ can not be rejected. In case normality and the white noise property of the errors $\epsilon_{t}$ in (2) are not invalidated, the adequacy of the restrictions $\mathrm{H}_{\mathrm{T}}$ can be tested with the conventional $F$ test statistic

$$
F_{\mathrm{T}}=((R R S S-U R S S)-1)((n-s k) /(s-1) k) \sim F((s-1) k, n-s k),
$$

where RRSS and URSS correspond to the residual sums of squares under the null hypothesis and under the unrestricted model, respectively. Note that model (1) essentially implies that a univariate time series model for $x_{i}$ is likely to be of the ARMA type. For convenience it is assumed in (2) that it can be approximated by an $\operatorname{AR}(k-1)$ model.

The restrictions in $\mathrm{H}_{\mathrm{T}}$ assume that all parameters in the dynamic model are equal. Somewhat less restricted hypotheses are

$$
\mathrm{H}_{\mathrm{RD}}: \alpha_{1 s}=\alpha_{s}, \ldots, \alpha_{k-1, s}=\alpha_{k-1} \text {, }
$$


i.e. the restricted dynamics hypothesis, of which the corresponding $F$ test, $F_{\mathrm{RD}}$, statistic follows an $F((s-1)(k-1), n-s k)$ distribution, and the restricted means hypothesis

$$
\mathrm{H}_{\mathrm{RM}}: \delta_{s}=\delta
$$

with its $F_{\mathrm{RM}}$ test statistic which follows an $F(s-1, n-s k)$ distribution. Additionally, one can test with $F$ tests the hypotheses that there are restricted means given the presence of restricted dynamics $\mathrm{H}_{\mathrm{RM} \| \mathrm{RD}}$, and vice versa.

Finally, to test the seasonal homoskedasticity hypothesis $\mathrm{H}_{\mathrm{SH}}$ in model (2), when it is restricted according to the outcomes of the above $F$ tests, one can estimate

$$
\hat{\epsilon}_{t}^{2}=\mu+\delta_{1} D_{1 t}+\delta_{2} D_{2 t}+\delta_{3} D_{3 t}+\kappa_{t}
$$

and test with an $F_{\mathrm{SH}}$ test the joint significance of the $\delta_{i}, i=1,2,3$. Rejection of one or more of the hypotheses $\mathrm{H}_{\mathrm{T}}, \mathrm{H}_{\mathrm{RD}}, \mathrm{H}_{\mathrm{RM}}, \mathrm{H}_{\mathrm{RM} \| \mathrm{RD}}$, and $\mathrm{H}_{\mathrm{SH}}$ indicates the presence of some type of seasonality.

\section{Examples}

The first example considers the 102 quarterly United Kingdom log stock prices series, in the period 1963.1 through 1988.4. An application of the Franses (1990) approach to model (1), where $p$ is set equal to 1 , indicates that $x_{t}=\Delta_{1} y_{t}$ is an appropriately transformed variable. The order of an adequate AR model for $x_{t}$ appears to be 1 , and hence $k$ in (2) is set equal to 2 . The estimated model also includes eight dummy variables for single observations to ensure normality of the residuals. The results for the $F$ type test statistics are reported in table 1. From the significance of $F_{\mathrm{RM}}$ and $F_{\mathrm{RT}}$ it is clear that the U.K. stock price series does not show seasonal patterns, in the sense that the mean of the first differenced series varies with the season.

The second example concerns the log of the quarterly U.S. CLI index series, measured in the period 1948.1-1987.4, and hence obtaining 160 data points. Again, the required differencing filter appears to be $\Delta_{1}$. A subset AR model with orders one and four is fitted, while normality is reached only after the removal of ten outlying observations. In this case $k$ is set equal to 3 , and $n$ equals 155. The test results are displayed in the third column of table 1 . They indicate that although the individual hypotheses $\mathrm{H}_{\mathrm{RD}}$ and $\mathrm{H}_{\mathrm{RM}}$ can not be rejected, the joint and conditional hypotheses are rejected. This suggests that the CLI index shows signs of a combined mean and dynamics type of

Table 1

Results of various $F$ tests for restrictions in a general unrestricted periodic autoregressive model.

\begin{tabular}{lll}
\hline Hypothesis a $^{\text {a }}$ & Stock prices & CLI index \\
\hline Restricted means and dynamics & $2.261^{* *}$ & $2.170^{* *}$ \\
Restricted means & $3.480 * *$ & 1.702 \\
Restricted dynamics & 0.625 & 1.482 \\
Restricted means given restricted dynamics & $3.930 * *$ & $3.317^{* *}$ \\
Restricted dynamics given restricted means & 0.964 & $4.630^{* *}$ \\
No seasonal heteroskedasticity & 1.550 & 0.537 \\
\hline
\end{tabular}

a Expressions for the hypotheses and the corresponding test statistics can be found in the text.

** Significant at a $5 \%$ level, when the test is assumed to be independent of the other $F$ tests. 
seasonality, or that cyclical and trend behavior vary per quarter. The latter finding supports the empirical evidence reported in Ghysels (1991).

\section{Conclusion}

The general-to-simple test procedure for seasonality proposed in this paper is easy to use. In fact, it seems appropriate for automatic application. Further, it yields a direct indication of the type of seasonal patterns. Compared to the tests proposed in Canova (1989), these seem to be its advantages.

An obvious disadvantage is that the individual test statistics are related, and hence that significance levels may need modification. Furthermore, ARCH effects and outliers complicate any straightforward model selection, and hence also tests for seasonality. How these issues affect the empirical performance of the proposed test procedure is yet to be investigated.

\section{References}

Canova, F., 1989, Seasonalities in foreign exchange markets, CentER discussion paper 891 (Tilburg University, Tilburg).

Franses, P.H., 1990, A multivariate approach to modeling univariate seasonal time series, Econometric Institute report $9101 /$ A. Submitted.

Ghysels, E., 1991, Are business cycle turning points uniformly distributed throughout the year?, Unpublished manuscript (University of Montreal, Montreal).

Johansen, S. and K. Juselius, 1990, Maximum likelihood estimation and inference on cointegration - with applications to the demand for money, Oxford Bulletin of Economics and Statistics 52, 169-210.

Osborn, D.R., 1991, The implications of periodically varying coefficients for seasonal time-series processes, Journal of Econometrics 48, 373-384. 